\title{
Diffraction in QCD
}

Boris Kopeliovich, Irina Potashnikova, and Ivan Schmidt

Departamento de Física, Universidad Técnica Federico Santa María, Casilla 110-V, Valparaíso, Chile

\section{Received on 20 November, 2006}

This lecture presents a short review of the main features of diffractive processes and QCD inspired models. It includes the following topics: (1) Quantum mechanics of diffraction: general properties; (2) Color dipole description of diffraction; (3) Color transparency; (4) Soft diffraction in hard reactions: DIS, Drell-Yan, Higgs production; (5) Why Pomerons interact weakly; (6) Small gluonic spots in the proton; (7) Diffraction near the unitarity bound: the Goulianos-Schlein "puzzle"; (8) Diffraction on nuclei: diffractive Color Glass; (9) CGC and gluon shadowing.

Keywords: Diffraction; QCD; Pomeron

\section{INTRODUCTION}

Diffraction is associated with the optical analogy, which is elastic scattering of light caused by absorption. A new feature of diffraction in quantum mechanics is the possibility of inelastic diffraction, which is nearly elastic scattering with excitation of one or both colliding hadrons to effective masses which are much smaller that the c.m. energy of the collision. The main bulk of diffractive events originate from soft interactions. Therefore, it is still a challenge to describe these processes starting from the first principles of QCD. Unavoidably, one faces the problem of confinement which is still a challenge for the theory. Nevertheless, the ideas of QCD help to develop quite an effective phenomenology for diffractive reactions, i.e. to establish relations between different observables. This lecture presents a mini-review of QCD based phenomenological models.

\section{NONABELIANCE AND DIFFRACTION}

Elastic and inelastic diffraction are large rapidity gap (LRG) processes. Since they emerge as a shadow of inelastic interactions, their amplitudes are nearly imaginary. This observation is a direct evidence for nonabeliance of the underlying theory.

Indeed, the elastic amplitude can be mediated only by a neutral exchange in t-channel, therefore the Born graphs in the abelian and nonabelian cases look like,

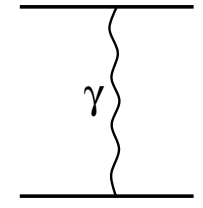

$\operatorname{Im~} \mathrm{f}_{\mathrm{el}}=0$

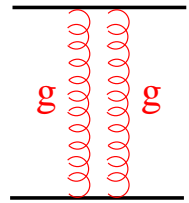

$\operatorname{Re} \mathrm{f}_{\mathrm{el}}=0$
FIG. 1: Born approximation for elastic scattering in abelian (left) and nonabelian (right) theories.

The striking difference between these two amplitudes is in their phases. In the abelian case (e.g. in QED) the Born amplitude is real, while in the nonabelian theory (QCD) the amplitude is imaginary.

Data for elastic hadron scattering show that the real part of the elastic amplitude is small, and this is a direct evidence for nonabeliance of the underlying dynamics. This is a remarkable observation, since we have known so far very few manifestations of nonabeliance in data.

The Born amplitude depicted in Fig. 1 is independent of energy. Gluon radiation gives rise to the energy dependence of the total cross section through the unitarity relation:

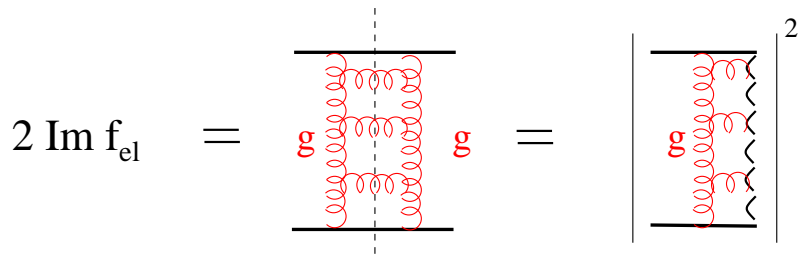

FIG. 2: The unitarity relation for the Pomeron amplitude in terms of perturbative QCD

Elastic scattering reaches maximal strength at the unitarity limit of black disc, $\operatorname{Im} f_{e l}(b)=1$,

$$
\sigma_{e l}=\sigma_{i n}=\pi R^{2}
$$

where $R$ is the radius of interaction.

The unitarity relation tells us that the imaginary part of the partial amplitude $\operatorname{Im} f_{e l}(b)$ cannot rise for ever. After the unitarity bound is reached, the total cross section can rise only due to an energy dependence of the interaction radius $R(s)$. Froissart theorem imposes a restriction on this, the interaction radius cannot rise with energy faster than $R \propto \ln (s)$. Then, the total and elastic cross section rise with energy as $\propto \ln ^{2}(s)$ in the Froissart regime of unitarity saturation.

\section{REGGE PHENOMENOLOGY}

In the Regge theory one assumes that the elastic amplitude is mediated by exchange of the rightmost singularity in the 
complex angular momentum plane. This singularity is called Pomeron.

The Regge trajectory corresponding to this singularity is approximately linear,

$$
\alpha_{\mathbb{P}}(t)=\alpha_{\mathbb{P}}^{0}+\alpha_{\mathbb{P}}^{\prime} t
$$

with parameters

$$
\begin{aligned}
\alpha_{\mathbb{P}}^{0} & =1.1 ; \\
\alpha_{\mathbb{P}}^{\prime} & =0.25 \mathrm{GeV}^{-2}
\end{aligned}
$$

This behavior follows from data for elastic and total cross sections fitted by the formula,

$$
f_{e l}(t)=\left[i-\operatorname{ctg} \frac{\pi \alpha_{P}(t)}{2}\right] h(t)\left(\frac{s}{s_{0}}\right)^{\alpha_{\mathbb{P}}(t)},
$$

where $h(t)$ is the phenomenological residue function which is not given by the theory, but is fitted to data. It correlates with the choice of the parameter $s_{0}$.

Apparently, the linear $t$-dependence of the Pomeron trajectory Eq. (3) cannot continue for ever at large negative $t$. Indeed, the higher order corrections in the ladder graph in Fig. 2 vanish as powers of the QCD coupling $\alpha_{s}(t)$ and the Pomeron trajectory $\alpha_{\mathbb{P}}(t)$ should approach the value corresponding to the Born graph, $\alpha_{P}(t) \rightarrow 1$. Indeed, the trajectory seems to level off at large $|t|$ according to data [1].

It has been a natural and simplest assumption made in the early years of the Regge theory that the Pomeron is a Regge pole with a linear trajectory and the intercept $\alpha_{I P}(t)=1$. Nowadays, however, we have a multi-choice answer, and it is still debatable whether the Pomeron is

- a Regge pole (probably not, since $\alpha_{\mathbb{P}}^{0}$ varies with $Q^{2}$ in DIS);

- or the DGLAP Pomeron [2], which corresponds to a specific ordering for radiated gluons in the ladder graph in Fig. $2, x \leq x_{i+1} \leq x_{i}$ and $k_{i+1}^{2}<k_{i}^{2} \leq Q^{2}$;

- or the BFKL Pomeron [3] which does not have ordering in transverse momenta of radiated gluons, but has no evolution with $Q^{2}$ either [4];

- or something else?

\section{A. Triple Regge phenomenology}

The cross section of the single-diffractive process, $a+b \rightarrow$ $X+b$ can be expressed in terms of the Regge approach. Indeed, if to sum up all final state excitations $X$, one can apply the unitarity relation to the Pomeron-hadron $(\mathbb{P}-a)$ amplitude as is shown in Fig. 3. Provided that the effective mass of the excitation is large (but not too much), $s_{0} \ll M_{X}^{2} \ll s$, one can describe the Pomeron-hadron elastic amplitude via exchange of the Pomeron or secondary Reggeons in the $t$-channel. One

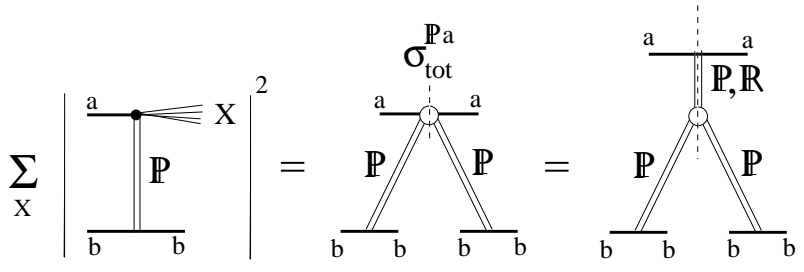

FIG. 3: The cross section of single diffraction, $a+b \rightarrow X+b$ summed over all excitation channels at fixed effective mass $M_{X}$

arrives to the triple-Regge graph, which corresponds to the cross section,

$$
\frac{d \sigma_{s d}^{a b \rightarrow X b}}{d x_{F} d t}=\sum_{i=\mathbb{P}, \mathbb{R}} G_{\mathbb{P} \mathbb{P} i}(t)\left(1-x_{F}\right)^{\alpha_{i}(0)-2 \alpha_{\mathbb{P}}(t)}\left(\frac{s}{s_{0}}\right)^{\alpha_{i}(0)-1}
$$

Here $x_{F}$ is the Feynman variable for the recoil particle $b, x_{F}=$ $2 p_{b}^{\|} / \sqrt{s} \approx 1-M_{X}^{2} / s$.

Equation (5) contains new phenomenological functions, effective triple-Regge vertices, $G_{I P I P I P}(t)$ and $G_{I P I P I R}(t)$. The diffractive cross section can also be expressed in terms of the Pomeron-hadron total cross section $\sigma_{\text {tot }}^{\mathbb{P a}}\left(s^{\prime}=M_{X}^{2}\right)$. Most interesting is the asymptotia $\left(s^{\prime}=M_{X}^{2} \gg s\right)$ of this cross section related to the triple-Pomeron coupling,

$$
G_{3 \mathbb{P}}(t)=\sigma_{t o t}^{\mathbb{P a} a} N_{\mathbb{P b b}}(t)^{2} \text {. }
$$

Here $N_{\mathbb{P} b b}(t)$ is the Pomeron - hadron vertex known from $b b$ elastic scattering. Thus, one can extract from data on single diffraction the Pomeron-hadron total cross section, $\sigma_{t o t}^{\mathbb{P a}}$, which carries unique information about the properties of the Pomeron (see below).

\section{QUANTUM MECHANICS OF DIFFRACTION}

Diffractive excitation is a nontrivial consequence of presence of quantum fluctuations in hadrons. In classical mechanics only elastic scattering is possible. An example is diffractive scattering of electromagnetic waves.

One can understand the origin of diffractive excitation in terms of elastic diffraction $[5,6]$. Since a hadron has a composite structure, different hadronic constituents interact differently causing a modification of the projectile coherent superposition of states. Such a modified wave packet is not orthogonal any more to other hadrons different from the incoming one. This makes possible production of new hadrons, i.e. diffractive excitation.

To simplify the picture, one can switch to the basis of eigenstates of the interaction. Since a hadron can be excited, it cannot be an eigenstate of the interaction, and can be expanded over the complete set of eigen states $|\alpha\rangle$ [7-9]:

$$
|h\rangle=\sum_{\alpha=1} C_{\alpha}^{h}|\alpha\rangle
$$

which satisfy the condition, $\hat{f}_{e l}|\alpha\rangle=f_{\alpha}|\alpha\rangle$, where $\hat{f}_{e l}$ is the elastic amplitude operator. 
Due to completeness and orthogonality of each set of states, the coefficient $C_{\alpha}^{h}$ in (7) satisfy the relations,

$$
\begin{aligned}
\left\langle h^{\prime} \mid h\right\rangle & =\sum_{\alpha=1}\left(C_{\alpha}^{h^{\prime}}\right)^{*} C_{\alpha}^{h}=\delta_{h h^{\prime}} \\
\langle\beta \mid \alpha\rangle & =\sum_{h^{\prime}}\left(C_{\beta}^{h^{\prime}}\right)^{*} C_{\alpha}^{h^{\prime}}=\delta_{\alpha \beta}
\end{aligned}
$$

The elastic and single diffraction amplitudes can be thus expressed via the eigen amplitudes as,

$$
\begin{aligned}
f_{e l}^{h \rightarrow h} & =\sum_{\alpha=1}\left|C_{\alpha}^{h}\right|^{2} f_{\alpha} \\
f_{s d}^{h \rightarrow h^{\prime}} & =\sum_{\alpha=1}\left(C_{\alpha}^{h^{\prime}}\right)^{*} C_{\alpha}^{h} f_{\alpha}
\end{aligned}
$$

Using these expressions and the completeness relations, Eqs. (8) one can calculate the forward single diffraction cross section without knowledge of the properties of $\left|h^{\prime}\right\rangle$,

$$
\begin{aligned}
\left.\sum_{h^{\prime} \neq h} \frac{d \sigma_{s d}^{h \rightarrow h^{\prime}}}{d t}\right|_{t=0} & =\frac{1}{4 \pi}\left[\sum_{h^{\prime}}\left|f_{s d}^{h h^{\prime}}\right|^{2}-\left|f_{e l}^{h h}\right|^{2}\right] \\
& =\frac{1}{4 \pi}\left[\sum_{\alpha}\left|C_{\alpha}^{h}\right|^{2}\left|f_{\alpha}\right|^{2}-\left(\sum_{\alpha}\left|C_{\alpha}^{h}\right| f_{\alpha}\right)^{2}\right] \\
& =\frac{\left\langle f_{\alpha}^{2}\right\rangle-\left\langle f_{\alpha}\right\rangle^{2}}{4 \pi}
\end{aligned}
$$

Thus, the forward diffractive cross section is given by the dispersion of the eigen values distribution. For some specific distributions the dispersion may be zero. For instance if all the eigen amplitudes are equal, or one of them is much larger than others.

According to Eqs. (9)-(10) one can calculate the total and diffractive cross sections on the same footing, provided that the eigenstates $|\alpha\rangle$, their weights $\left|C_{\alpha}^{h}\right|^{2}$ and the eigenvalues $f_{\alpha}$ are known. Notice that the eigen amplitudes $f_{\alpha}$ are the same for different hadronic species $|h\rangle$. This remarkable property of eigen amplitudes is employed later on.

In the Froissart regime all the partial eigen amplitudes reach the unitarity limit, $\operatorname{Im} f_{\alpha}=1$. Then, according to the completeness conditions,

$$
\begin{aligned}
f_{e l}^{h h} & \Rightarrow \sum_{\alpha=1}\left|C_{\alpha}^{h}\right|^{2}=1 \\
f_{s d}^{h h^{\prime}} & \Rightarrow \sum_{\alpha=1}\left(C_{\alpha}^{h^{\prime}}\right)^{*} C_{\alpha}^{h}=0
\end{aligned}
$$

Diffraction is impossible within a black disc, but only on its periphery, $b \sim R$. Since in the Froissart regime $R \propto \ln (s)$,

$$
\begin{aligned}
\sigma_{t o t} & \propto \sigma_{e l} \propto \ln ^{2}(s) \\
\sigma_{s d} & \propto \ln (s),
\end{aligned}
$$

i.e. $\sigma_{s d} / \sigma_{t o t} \propto 1 / \ln (s)$.

\section{LIGHT-CONE COLOR DIPOLE DESCRIPTION}

The choice of the eigen state basis depends on the underlying theory. It was first realized in [10] that the eigenstates of interaction in QCD are color dipoles. Such dipoles cannot be excited and can experience only elastic scattering. Indeed, high energy dipoles have no definite mass, but only separation $\vec{r}_{T}$ which cannot be altered during soft interaction. The eigenvalues of the total cross section, $\sigma\left(r_{T}\right)$, also depend on $r_{T}$, but may also depend on energy.

The total and single diffractive cross sections read,

$$
\begin{aligned}
\sigma_{\text {tot }}^{h p} & =\sum_{\alpha=1}\left|C_{\alpha}^{h}\right|^{2} \sigma_{\alpha} \\
& =\int d^{2} r_{T}\left|\Psi_{h}\left(r_{T}\right)\right|^{2} \sigma\left(r_{T}\right)=\left\langle\sigma\left(r_{T}\right)\right\rangle ;
\end{aligned}
$$

and

$$
\begin{aligned}
& \left.\sum_{h^{\prime}} \frac{d \sigma_{s d}^{h \rightarrow h^{\prime}}}{d t}\right|_{t=0}=\sum_{\alpha=1}\left|C_{\alpha}^{h}\right|^{2} \frac{\sigma_{\alpha}^{2}}{16 \pi}= \\
& \int d^{2} r_{T}\left|\Psi_{h}\left(r_{T}\right)\right|^{2} \frac{\sigma^{2}\left(r_{T}\right)}{16 \pi}=\frac{\left\langle\sigma^{2}\left(r_{T}\right)\right\rangle}{16 \pi} .
\end{aligned}
$$

The eigenvalue of the cross section for a simplest $\bar{q} q$ dipole $\sigma_{\bar{q} q}\left(r_{T}\right)$ is a fundamental flavor independent quantity. Its calculation is still a theoretical challenge, but it can be fitted to data.

A rich source of information about $\sigma_{\bar{q} q}\left(r_{T}\right)$ is DIS. At small $x_{B j}$ the virtual photon exposes hadronic properties as is illustrated in Fig. 4.
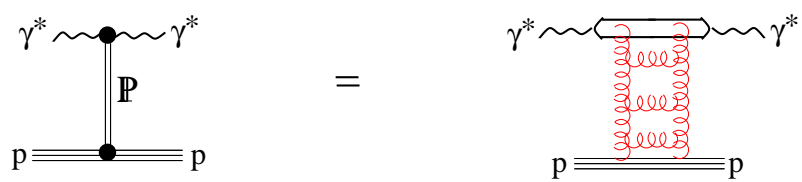

FIG. 4: The virtual photon interacts via its hadronic fluctuations which are $\bar{q} q$ dipoles and more complicated Fock states. The Pomeron exchange is illustrated as a perturbative ladder.

One has a control of the dipole size varying the photon virtuality $Q^{2}$ according to the factorized formula $[10,11]$

$$
\sigma_{\text {tot }}^{\gamma^{*} p}\left(Q^{2}, x_{B j}\right)=\int d^{2} r_{T} \int_{0}^{1} d x\left|\Psi_{\gamma^{*}}\left(r_{T}, Q^{2}\right)\right|^{2} \sigma_{\bar{q} q,}\left(r_{T}, x_{B j}\right)
$$

One may expect, both intuitively and considering dimensions, that the mean transverse separation is $\left\langle r_{T}^{2}\right\rangle \sim 1 / Q^{2}$. However, the situation is more complicated than that.

\section{A. The photon distribution amplitudes}

The dipole size in (15) is governed by the photon $\bar{q} q$ lightcone wave function $\Psi_{\gamma^{*}}\left(r_{T}, \alpha, Q^{2}\right)$ where $\alpha$ is the fraction of the photon light-cone momentum carried by the quark as is illustrated in Fig. 5. This wave function can be calculated perturbatively [12].

$$
\Psi_{\gamma^{*}}^{T, L}\left(\vec{r}_{T}, \alpha\right)=\frac{\sqrt{\alpha_{e m}}}{2 \pi} \bar{\chi} \widehat{O}^{T, L} \chi K_{0}\left(\varepsilon r_{T}\right)
$$




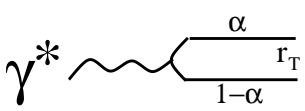

FIG. 5: Photon virtual dissociation to a $\bar{q} q$ pair with transverse separation $r_{T}$ and sharing of the light-cone momentum $\alpha$ and $1-\alpha$.

where $\varepsilon^{2}=\alpha(1-\alpha) Q^{2}+m_{q}^{2}$;

$$
\begin{aligned}
& \widehat{O}^{T}=m_{q} \vec{\sigma} \vec{e}+i(1-2 \alpha)(\vec{\sigma} \vec{n})\left(\vec{e} \vec{\nabla}_{r_{T}}\right)+(\vec{\sigma} \times \vec{e}) \vec{\nabla}_{r_{T}} ; \\
& \widehat{O}^{L}=2 Q \alpha(1-\alpha) \vec{\sigma} \vec{n}
\end{aligned}
$$

It might be confusing that these wave functions are not normalized, the transverse part is even divergent. Therefore it is better to call them distribution amplitudes.

The mean transverse $\bar{q} q$ separation for a transversely polarized photon is,

$$
\left\langle r_{T}^{2}\right\rangle \sim \frac{1}{\varepsilon^{2}}=\frac{1}{Q^{2} \alpha(1-\alpha)+m_{q}^{2}},
$$

i.e. the separation is about as small as $1 / Q^{2}$, except the endpoints $\alpha \rightarrow 0$ or 1 . Notice that $m_{q} \sim \Lambda_{Q C D}$ plays here the role of an infra-red cut off.

\section{B. Dipole cross section and color transparency}

The central ingredient of Eq. (15) is the phenomenological universal cross section $\sigma_{\bar{q} q}\left(r_{T}, x\right)$ for the interaction of a nucleon with a $\bar{q} q$ dipole of transverse separation $r_{T}$. It must me energy dependent due to the higher-order corrections shown in Fig. 4. In the presence of hard scale the dimensionless quantity must be $s / Q^{2}=1 / x$ where $x$ is the Bjorken variable. The parametrization suggested in [13],

$$
\sigma_{\bar{q} q}\left(r_{T}, x\right)=\sigma_{0}\left[1-e^{-\frac{1}{4} r_{T}^{2} Q_{s}^{2}(x)}\right],
$$

successfully fits HERA data for the proton structure function $F_{2}\left(x, Q^{2}\right)$ at small $x$ with parameters: $Q_{s}(x)=1 \mathrm{GeV} \times$ $\left(x_{0} / x\right)^{\lambda / 2}$ and $\sigma_{0}=23.03 \mathrm{mb} ; \lambda=0.288 ; x_{0}=3.04 \cdot 10^{-4}$. This cross section incorporates the phenomenon of saturation at a soft scale, since it levels off at large separations, $r_{T}^{2} \gg 1 / Q_{s}^{2}$.

A remarkable feature of this dipole cross section is Color Transparency (CT), namely for small dipoles, $r_{T} \rightarrow 0$, the cross section vanishes as $\sigma_{\bar{q} q}\left(r_{T}\right) \propto r_{T}^{2}$ [10]. This is a much more general property of any dipole cross section in QCD, since a point-like colorless object cannot interact with external color fields. The quadratic $r_{T}$-dependence is a direct consequence of gauge invariance and nonabeliance of QCD.

The effect of CT has been searched for in different reactions. In some of them, quasielastic high- $p_{T}$ scattering of electrons [14] and hadrons [15], no unambiguous signal of CT was observed. Those processes turned out to be unsuitable for CT searches [16, 17], since the formation length of the hadrons was too short compared to the nuclear size.
More successful was search for CT in diffractive leptoproduction of vector mesons, proposed in [18] and confirmed by the E665 experiment [19]. This process illustrated in Fig. 6 is different from DIS by the projection of the produced $\bar{q} q$ dipole on the vector meson wave function. This projection

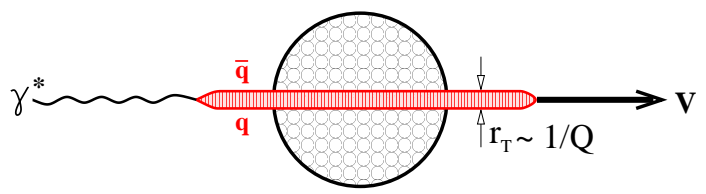

FIG. 6: Quasielasic virtual photoproduction of vector mesons. At high $Q^{2}$ the $\bar{q} q$ dipole experiences little attenuation in the nucleus.

suppresses the endpoint part of the distribution amplitude and makes the signal of CT stronger. A new measurement of the effect of CT was done recently by the HERMES experiment [20]. They found a good signal of CT in accordance with theoretical predictions [21].

Another diffractive process suggested in [22], coherent production of high- $p_{T}$ back-to-back jets on nuclei, also revealed a strong signal of CT [23] in good agreement with theoretical estimates [24]. In this process the nucleus remains intact, which is possible due to sufficiently high energy. A bright signature of CT observed in the E791 experiment at Fermilab is the $A$-dependence of the coherent cross section, $\propto A^{4 / 3}$. This corresponds to full nuclear transparency.

\section{SOFT DIFFRACTION IN HARD REACTIONS}

\section{A. Diffractive DIS}

The contribution of diffractive quasielastic production of vector mesons (see Fig. 6) is a tiny fraction, vanishing as $1 / Q^{2}$, of the total inclusive DIS cross section. However the fraction of all diffractive events associated with large rapidity gaps in DIS is large, about $10 \%$, and is nearly independent of $Q^{2}$. It turns out to be a result of a contribution of rare soft fluctuations in the hard photon. According to (18) a longitudinally asymmetric $\bar{q} q$ pair with $\alpha$ or $1-\alpha \sim 1 / Q^{2}$ have a large hadronic size and experience soft diffractive interactions like hadrons. Although the admixture of such soft fluctuations in the virtual photon is tiny, that may be compensated by a large interaction cross section. This interplay between the fluctuation probability and the cross section is illustrated for inclusive and diffractive DIS in Table. 1 [25]. Hard fluctuations of the photon have large weight, but vanishing as $1 / Q^{2}$ cross section, while soft fluctuations have a small, $m_{q}^{2} / Q^{2}$, weight, but interact strongly, $\sigma \sim 1 / m_{q}^{2}$. The latter factor compensates the smallness of the probability in the case of DIS, and overcompensates it for diffraction.

Thus, we conclude that inclusive DIS is semi-hard and semi-soft, and the soft component is present at any high $Q^{2}$. On the other hand, diffractive DIS (called sometimes "hard diffraction") is predominantly a soft process. This is why its fraction in the total DIS cross section is nearly $Q^{2}$ - 
TABLE I: Interplay between the probabilities of hard and soft fluctuations in a highly virtual photon and the cross section of interaction of these fluctuations.

\begin{tabular}{|c|c|c|c|c|}
\hline & $\left|C_{\alpha}\right|^{2}$ & $\sigma_{\alpha}$ & $\sigma_{\text {tot }}=\sum_{\alpha=\text { soft }}^{\text {hard }}\left|C_{\alpha}\right|^{2} \sigma_{\alpha}$ & $\sigma_{\text {sd }}=\sum_{\alpha=\text { soft }}^{\text {hard }}\left|C_{\alpha}\right|^{2} \sigma_{\alpha}^{2}$ \\
\hline Hard & $\sim 1$ & $\sim \frac{1}{Q^{2}}$ & $\sim \frac{1}{Q^{2}}$ & $\sim \frac{1}{Q^{4}}$ \\
\hline Soft & $\sim \frac{m_{q}^{2}}{Q^{2}}$ & $\sim \frac{1}{m_{q}^{2}}$ & $\sim \frac{1}{Q^{2}}$ & $\sim \frac{1}{m_{q}^{2} Q^{2}}$ \\
\hline
\end{tabular}

independent. One can test this picture studying the $Q^{2}$ dependence of the diffractive DIS [26].

Since diffraction is a source of nuclear shadowing [27], it also should scale in $x$. Indeed, most of experiment have not found any variation with $Q^{2}$ of shadowing in DIS on nuclei. Only the NMC experiment managed to find a weak scaling violation which agrees with theoretical expectations [28].

Notice that in spite of independence of $Q^{2}$, both diffraction and shadowing are higher twist effects. This is easy to check considering photoproduction of heavy flavors. In this case the hard scale is imposed by the heavy quark mass, and diffraction becomes a hard process with cross section vanishing as $1 / m_{Q}^{4}$. Nuclear shadowing also vanishes as $1 / m_{Q}^{2}$.

The true leading twist diffraction and shadowing are associated with gluon radiation considered below.

\section{B. Diffractive Drell-Yan reaction}

The dipole description of the Drell-Yan reaction in many respects is similar to DIS. This is not a surprize, since the two processes are related by QCD factorization. The cross section of heavy photon $\left(\gamma^{*} \rightarrow \overline{l l}\right)$ radiation by a quark reads [29-32],

$$
\frac{d \sigma\left(q p \rightarrow \gamma^{*} X\right)}{d \ln \alpha}=\int d^{2} r_{T}\left|\Psi_{\gamma^{*} q}^{T, L}\left(\alpha, r_{T}\right)\right|^{2} \sigma_{q \bar{q}}\left(\alpha r_{T}, x\right),
$$

Here $\alpha$ is the fraction of the quark light-cone momentum taken away by the dilepton; $r_{T}$ is the photon-quark transverse separation; and the light-cone distribution function $\Psi$ is similar to one in DIS, Eq. (16), and can be found in [29-31].

Notice that the dileptons are radiated only in the fragmentation region of the quark and are suppressed at mid rapidities. Indeed, due to $\mathrm{CT}$ the dipole cross section vanishes as $\sigma_{q \bar{q}}\left(\alpha r_{T}, x\right) \propto \alpha^{2}$ at $\alpha \rightarrow 0$.

There is an important difference between DIS and DY reaction. In the inclusive DIS cross section one integrates over $0<\alpha<1$, this is why this cross section is always a mixture of soft and hard contributions (see Table 1). In the case of DY reaction there is a new variable, $x_{1}$, which is fraction of the proton momentum carried by the dilepton. Since $\alpha>x_{1}$, one can enhance the soft part of the DY cross section selecting events with $x_{1} \rightarrow 1$. This soft part of the DY process is subject to unitarity corrections [33] which are more important than in DIS [34].
Another distinction between DIS and DY is the suppression of the DY diffractive cross section. Namely, the forward cross section of diffractive radiation $q p \rightarrow \bar{l} l q p$ is zero [30]. Indeed, according to (10) the forward diffractive cross section is given by the dispersion of the eigen amplitude distribution. However, in both eigen states $|q\rangle$ and $\left|q \gamma^{*}\right\rangle$ only the quark interacts. So the two eigen amplitudes are equal, and the dispersion is zero.

Nevertheless, in the case of hadronic collision diffractive DY cross section does not vanish in the forward direction. In this case the two eigen states are $|\bar{q} q\rangle$ and $\left|\bar{q} q \gamma^{*}\right\rangle$ (for the sake of simplicity we take a pion). The interacting component of these Fock states is the $\bar{q} q$ dipole, however it gets a different size after the $q$ or $\bar{q}$ radiates the photon. Then the two Fock states interact differently, and this leads to a nonvanishing forward diffraction. Notice that the diffractive cross section is proportional to the dipole size [35].

\section{Diffractive Higgs production}

Diffractive higgsstrahlung is rather similar to diffractive DY, since in both cases the radiated particle does not take part in the interaction [35]. However, the Higgs coupling to a quark is proportional to the quark mass, therefore, the cross section of higgsstrahlung by light hadrons is vanishingly small.

A larger cross section may emerge due to admixture of heavy flavors in light hadrons. A novel mechanism of exclusive Higgs production, $p p \rightarrow H p p$, due to direct coalescence of heavy quarks, $\bar{Q} Q \rightarrow H$ was proposed in [36]. The cross section of Higgs production was evaluated assuming $1 \%$ of intrinsic charm (IC) [37] and that heavier flavors scale as $1 / \mathrm{m}_{Q}^{2}$ [38]. The results are shown in Fig. 7 as a function of Higgs mass for different intrinsic heavy flavors.

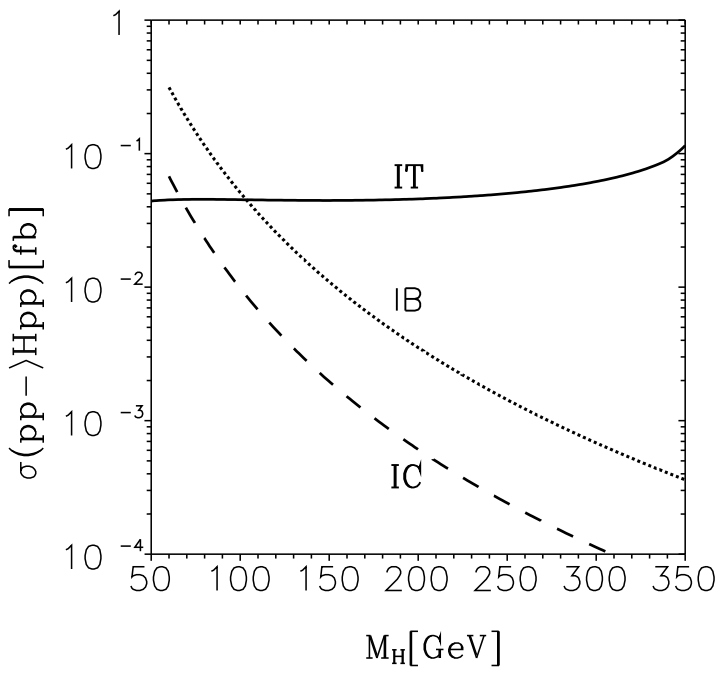

FIG. 7: Cross section of exclusive diffractive Higgs production, $p p \rightarrow H p p$, from intrinsic charm (IC), bottom (IB) and top (IT) [36]. 


\section{DIFFRACTIVE EXCITATION OF HADRONS}

\section{A. Excitation of the valence quark skeleton}

A hadron can be excited in diffractive reaction $h p \rightarrow X p$ by different mechanisms. One possibility is to excite the valence quark skeleton without gluon radiation $[39,40]$. This process is illustrated in Fig. 8 A clear signature of this process is the

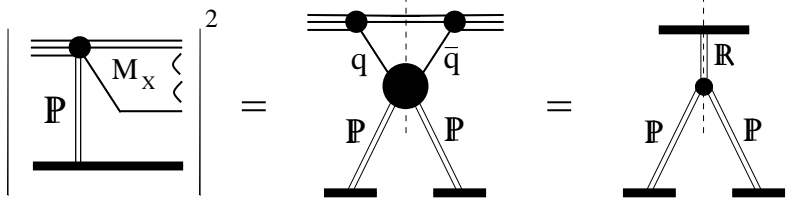

FIG. 8: Diffractive excitation of the valence quark skeleton of a hadron.

dependence of the cross section on effective mass of the excitation, $M_{X}$. It must be $d \sigma_{s d} / d M_{X}^{2} \propto 1 / M_{X}^{3}$, since is related to the intercept of the secondary Reggeon, $\alpha_{\mathbb{R}}(0)=1 / 2$, as is demonstrated in Fig. 8.

The specific mass-dependence allows to single out this contribution from data for diffractive reaction $p p \rightarrow p X$, Using the results of the triple-Regge analysis of data [41] we can evaluate the relative probability of excitation with no gluon radiation [40],

$$
R_{s d}=\left.\frac{d \sigma_{s d} / d p_{T}^{2}}{d \sigma_{e l} / d p_{T}^{2}}\right|_{p_{T}=0}=\frac{5.5 \mathrm{mb} / \mathrm{GeV}^{2}}{84.5 \mathrm{mb} / \mathrm{GeV}^{2}}=0.065
$$

This fraction turns out to be very small, only few percent from the forward elastic cross section. This suppression can be understood as follows. In terms of duality the tripleRegge graph in Fig. 8 is equivalent to excitation of nucleon resonances. Their and proton wave functions are orthogonal. Therefore the matrix element $\left\langle\Psi_{p}\left(r_{T}\right)\left|\sigma\left(r_{T}\right)\right| \Psi_{X}\left(r_{T}\right)\right\rangle$ is not zero only due to variation of the dipole cross section with $r_{T}$. However, since the dipole cross section levels off at large separations, only the short range part of the integration, $r_{T}<1 / Q_{s} \sim 0.3 \mathrm{fm}$ contributes to the overlap integral. This explains the observed smallness of gluonless diffraction [40].

\section{B. Diffractive gluon radiation}

A hadron can be excited differently, by shaking off a part of its gluonic field in the form of gluon radiation. Since gluons are vector particles, they can propagate through large rapidity intervals without attenuation and carry a tiny fraction of the hadron momentum. Therefore the effective mass of the excitation should be large. This is a decisive signature of radiation, the high-mass tail of the diffractive cross section, $d \sigma_{s d} / d M_{X}^{2} \propto 1 / M_{X}^{2}$. Observation of this behavior is undebatable proof for excitation via gluon radiation, and the cross section can reliably singled out of data [41].

Fig. 9 shows how the cross section of diffractive gluon radiation is related to the triple-Pomeron graph. According to
Eq. (6) it can also be expressed in terms of the Pomeronproton total cross section $\sigma_{\text {tot }}^{\mathbb{P} p}\left(M_{X}^{2}\right)$, for which the effective mass of the excitation plays role of the c.m. energy.

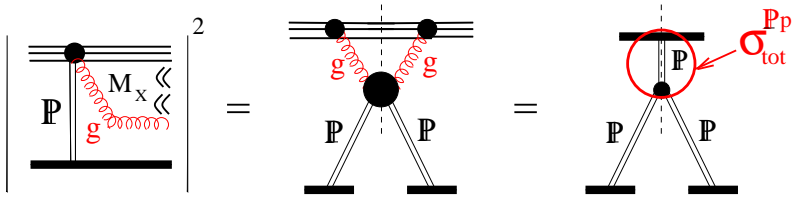

FIG. 9: The cross section of diffractive gluon radiation related to the triple-Pomeron term, or to the Pomeron-proton total cross section.

The triple-Regge fit to data [41] with parametrization (5) reliably fixes the triple-Pomeron term which provides unique information about $\sigma_{\text {tot }}^{\mathbb{P} p}$. Since the Pomeron is a gluonic colorless dipole, one should probably expect a cross section about $9 / 4$ times larger than for mesons. Therefore, one expects $\sigma_{\text {tot }}^{I P p} \sim 50 \mathrm{mb}$.

Surprisingly, data analyses [42-44] lead to

$$
\sigma_{\text {tot }}^{\mathbb{P p}} \sim 2 \mathrm{mb}(!)
$$

\section{Small gluonic spots}

Why does the Pomeron interact so weakly, while gluons interact stronger than quarks?

One should recall color transparency: the Pomeron-proton cross section vanishes if the transverse size of the Pomeron (gluonic dipole) is small.

This effect of smallness of gluonic dipoles cannot be explained in pQCD which treats quarks and gluons as free particles. This is a nonperturbative phenomenon which may be related to the small size of gluonic fluctuations in the instantonliquid model [45]. It is also supported by calculations on the lattice [46] which reveal a very short gluon correlation radius.

The shape of the impact-parameter distribution of gluons is not known, but important is the mean size of the dipole. This size $r_{0}$ treated as a phenomenological parameter was fixed at $r_{0}=0.3 \mathrm{fm}$ by a fit to diffraction data [9]. Thus, we arrive at an image of the proton shown in Fig. 10. Gluons in the

FIG. 10: Light-cone snap-shot of the proton.

proton are located in small spots of size $0.3 \mathrm{fm}$. We employ this picture in what follows and provide more evidence for it.

\section{TOTAL AND ELASTIC CROSS SECTIONS}

Presence of the semihard scale $r_{0}$ allows to use pQCD to calculate in a parameter-free way the cross section of gluon 
bremsstrahlung rising with energy. The calculations performed in [47] confirm this.

\section{A. Total cross section}

The hadronic cross section was found in $[47,48]$ to have the following structure,

$$
\sigma_{t o t}=\sigma_{0}+\sigma_{1}\left(\frac{s}{s_{0}}\right)^{\Delta} .
$$

Here $\sigma_{0}$ is the term related to hadronic collisions without gluon radiation. In the string model, for instance, it corresponds to string crossing and flipping. This part of the cross section is independent of energy, since is related to the Lorentz invariant transverse size of the quark skeleton.

The second term in (2) is related to the contribution of gluon bremsstrahlung to the total cross section. Since this part is expected to be as small as $r_{0}^{2}, \sigma_{1}$ should be small either. Indeed, it was found in [47] that $\sigma_{1}=27 / 4 C r_{0}^{2}$, where the factor $C \approx 2.4$ is related to the behavior at small separations of the dipole-proton cross section calculated in Born approximation, $\sigma\left(r_{T}\right)=C r_{T}^{2}$ at $r_{T} \rightarrow 0$.

The energy dependence of the second term in (23) was found to be rather steep,

$$
\Delta=\frac{4 \alpha_{s}}{3 \pi} \approx 0.17
$$

This exponent seems to be too large compared to the experimentally measured $\sigma_{t o t} \propto s^{\varepsilon}$ with $\varepsilon \approx 0.1$. Nevertheless, formula (23) describe data well as is shown in Fig. 11. This is not a surprise, the energy dependence is slowed down by presence of the first energy independent term in (23). If to approximate the cross section (23) by the simple power dependence $s^{\varepsilon}$, then the effective exponent reads,

$$
\varepsilon=\frac{\Delta}{1+\sigma_{0} / \sigma_{1}\left(s / s_{0}\right)^{-\Delta}}
$$

So, one should expect the steepness of the energy dependence of the total cross section to rise with energy.

\section{B. Elastic slope}

The mean size, $\left\langle r^{2}(s)\right\rangle$, of the gluonic spots (Fig. 10) rises with energy due to Brownian motion performed by multiply radiated gluons. The speed of this growth is related to the slope parameter, $\alpha_{I P}^{\prime}$ of the Pomeron trajectory,

$$
\frac{1}{4} \frac{d\left\langle r^{2}(s)\right\rangle}{d \ln (s)}=\alpha_{I P}^{\prime}=\frac{\alpha_{s}}{3 \pi} r_{0}^{2}=0.1 \mathrm{GeV}^{-2}
$$

In the Regge approach this phenomenon is related to the $t$-slope of the differential elastic cross section, $B_{e l}(s)=B_{0}+$ $2 \alpha_{I P}^{\prime} \ln (s)$.

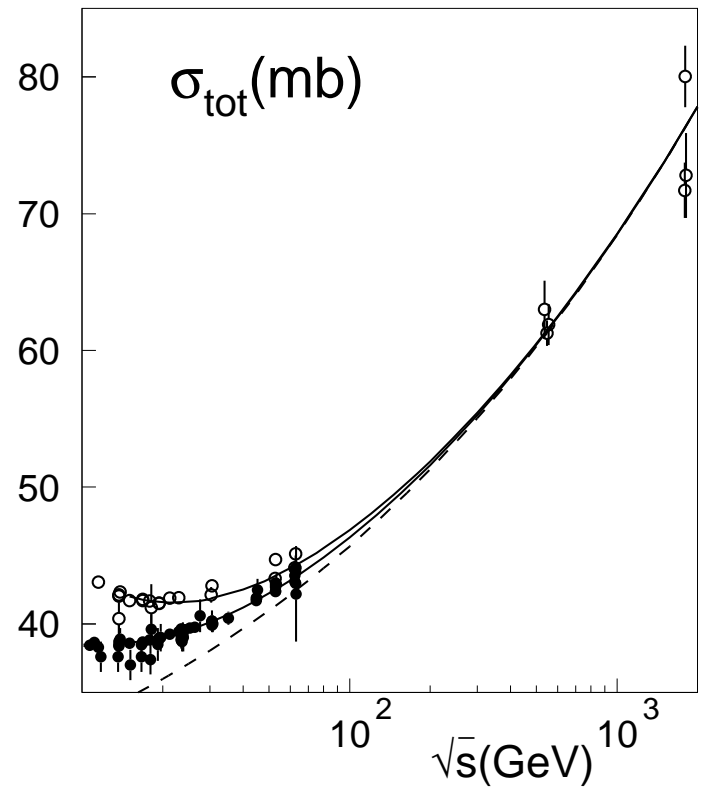

FIG. 11: Total $p p$ (closed points) and $\bar{p} p$ (open points) cross sections. Dashed curve is the Pomeron contribution Eq. (23) with one parameter $\sigma_{0}$ adjusted to a single experimental point at $\sqrt{s}=$ $540 \mathrm{GeV}$, other parameters are calculated. Solid curves include Reggeons which are fitted to data to describe the cross section at low energies.

Using (26) and data for electromagnetic proton formfactor to calculate $B_{0}$, the energy dependent elastic slope was calculated in [47] in good agreement with data shown in Fig. 12. Notice that the amplitude, Eq. (23), was unitarized in these calculations what is important for $p p$ scattering (see next section).

\section{SATURATION OF THE UNITARITY BOUND}

The mean number of radiated gluons slowly rises with energy, as well as the mean size of the gluonic clouds in Fig. 10. This gives rise to an energy dependence of the interaction radius in accordance with (26).

However, the predicted value (26) of parameter $\alpha_{\mathbb{P}}^{\prime}$ is rather small compared to the effective one, known from $p p$ data, $\alpha_{\text {eff }}^{\prime}=0.25 \mathrm{GeV}^{-2}$. Why does the slope shown in Fig. 12 rise so steeply with energy? Does it contradict theoretical expectations?

\section{A. Onset of the Froissart regime}

There is another source of energy dependence of the interaction radius related to the closeness of the unitarity bound. The rise of the total cross section can originate either from the rise of the partial amplitude, or from increase of the interaction radius. In the vicinity of the unitarity bound, $\operatorname{Im} f_{e l}(b) \leq$ 1 , the partial amplitude cannot rise any more in central collisions, while on the periphery the amplitude is small and there 


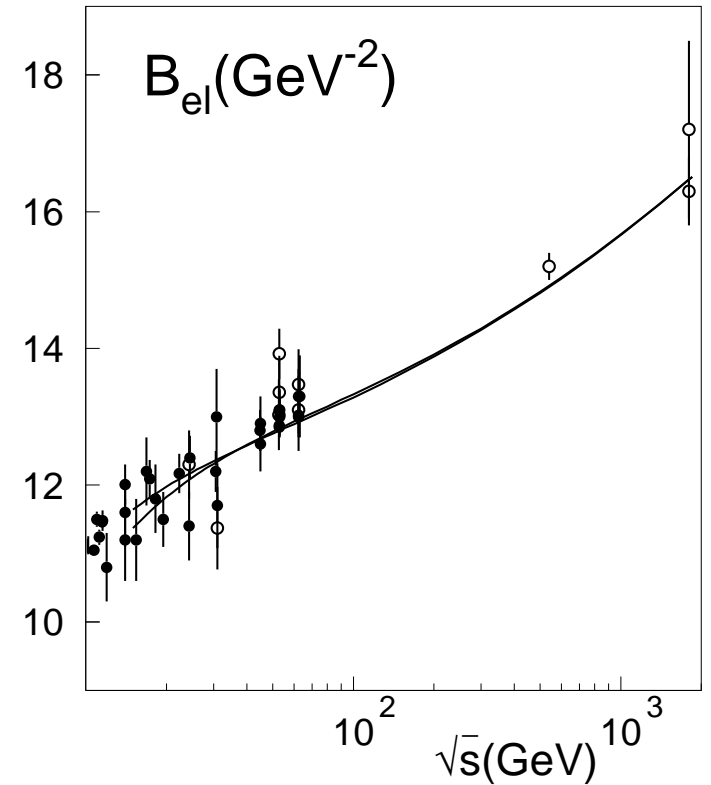

FIG. 12: Slope parameter for $p p$ (closed points) and $\bar{p} p$ (open points) elastic cross sections. Solid curves include Reggeons which fitted to data for total cross section (Fig. 11).

is still room for growth. This is how the interaction radius rises $[47,48]$.

In the Froissart regime (unitarity saturation) the interaction area rises $\propto \ln ^{2}(s)$ resulting in a fast shrinkage of the diffraction cone. In the regime of saturation $\alpha_{e f f}^{\prime} \gg \alpha_{I P}^{\prime}$. Apparently, an onset of this phenomenon explains why the elastic slope, $B_{e l}=\frac{1}{2}\left\langle b^{2}\right\rangle$, rises with energy much steeper than is predicted by (26).

In order to figure out whether the unitarity limit is indeed reached in $p p$ collisions at high energies, one can explicitly check with the partial amplitude related via Fourier transformation to the measured elastic differential cross section. This is depicted in Fig. 13. Indeed, both the data and theory demonstrate a nearly-saturation of the unitarity bound for central $p p$ collisions.

\section{B. Far from saturation: $J / \Psi$ production}

In order to test whether a substantial part of the observed $\alpha_{\text {eff }}^{\prime}$ indeed originates from saturation, one should look at a diffractive reaction for which the partial amplitude is far from the unitarity bound. Then one should expect the parameter $\alpha_{e f f}^{\prime}$ to get its genuine value $\alpha_{e f f}^{\prime}=\alpha_{I P}^{\prime}=0.1 \mathrm{GeV}^{-2}$ without absorptive (unitarity) corrections [49].

$J / \Psi$-proton elastic scattering would be very suitable for this purpose. Indeed, the partial amplitude for central collision $(b=0)$ can be evaluated as,

$$
f_{e l}^{\Psi p}(0)=\frac{\sigma_{t o t}^{\Psi p}}{4 \pi B_{e l}^{\Psi p}}=0.3 .
$$

For this estimate we assumed the energy range of HERA $\sqrt{s} \sim$
$\operatorname{Im} \Gamma(\mathrm{b})$

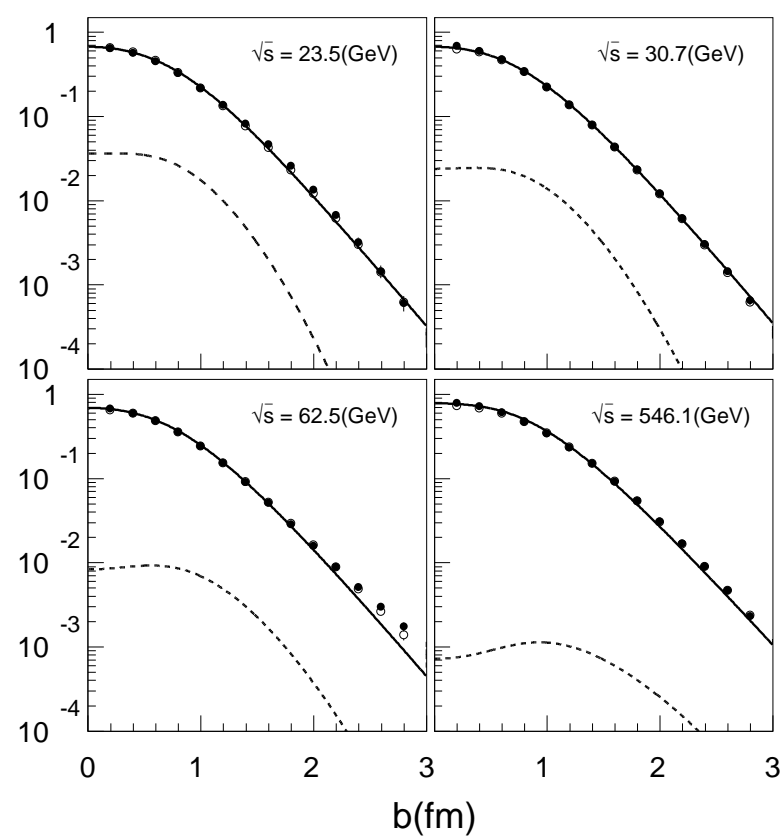

FIG. 13: Imaginary part of the partial elastic amplitude as function of impact parameter. Points are the Fourier transformed experimental data for differential cross section [47]. Solid curves show the results of calculation with the unitarized amplitude Eq. (23). Dashed curves show the contribution of the secondary Reggeons strongly shadowed by the unitarity corrections (see [47] for details).

$100 \mathrm{GeV}$ and used $\sigma_{\text {tot }}^{\Psi p}=6 \mathrm{mb}[50]$ and $B_{e l}^{\Psi p}=4 \mathrm{GeV}^{-2}$ [51].

Thus, the partial amplitude for $J / \Psi$-proton elastic scattering is safely quite below the unitarity bound at all impact parameters. Then one should observe a reduced value of $\alpha_{e f f}^{\prime}$ in accordance with (26).

Of course $J / \Psi$-proton scattering is not accessible, but it can be replaced by elastic photoproduction, $\gamma p \rightarrow J / \Psi p$, having in mind vector dominance. Although vector dominance is a poor approximation for charmonia [52], this only enforces our statements, since the real $\bar{c} c$ dipole is smaller than $J / \Psi$.

The measurements of energy dependent $t$-slope of the photoproduction cross section was performed by ZEUS collaboration at HERA. The result for the Pomeron trajectory are shown in Fig. 14.

These data fitted with $\alpha_{\mathbb{P}}(t)=\alpha_{\mathbb{P}}^{0}+\alpha_{\mathbb{P}}^{\prime} t$ result in,

$$
\begin{aligned}
& \alpha_{I P}^{0}=0.2 \pm 0.009 \\
& \alpha_{\mathbb{P}}^{\prime}=0.115 \pm 0.018 \mathrm{GeV}^{-2} .
\end{aligned}
$$

The value of $\alpha_{\mathbb{P}}^{\prime}$ is in good agreement with the prediction (26) made in [47].

\section{Goulianos-Schlein puzzle}

The unitarity or absorptive corrections are especially significant for off-diagonal diffractive channels. As was demon- 
ZEUS

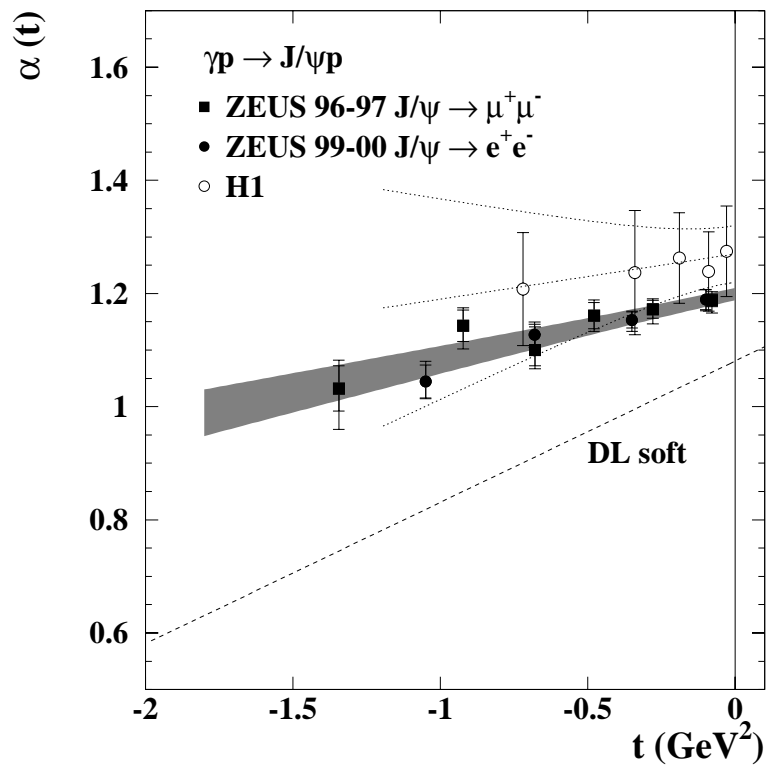

FIG. 14: The Pomeron trajectory $\alpha_{I P}(t)$ for elastic photoproduction of $J / \Psi$ measured at $\sqrt{s}=35-350 \mathrm{GeV}$ [51]. the solid line is a fit (see text).

strated in (11) diffraction is completely terminated in the unitarity limit. As far as we already have an onset of the Froissart regime, diffraction has to be suppressed, and the higher the energy is, the more.

The absorptive corrections to the diffractive amplitude have the form

$$
f_{s d}(b) \Rightarrow f_{s d}(b)\left[1-\operatorname{Im} f_{e l}(b)\right]
$$

Thus, at the unitarity bound, $\operatorname{Im} f_{e l}(b) \rightarrow 1$, diffraction vanishes everywhere except the very periphery.

Do we see any suppression in data?

Yes, a strong deviation from the nonunitarized Regge model was found in [43, 44] for single diffraction. Comparison of available data with the triple-Regge prediction uncorrected for unitarity, is shown in Fig. 15.

Notice that the effect of unitarity corrections Eq. (18) cannot be reproduced by a simple suppression of the Pomeron flux. The flux damping or renormalization factor is independent of impact parameter, while this dependence is the central issue in (18) (see in [54]).

\section{DIFFRACTIVE COLOR GLASS}

Nuclear targets allow to access the unitarity bound at much lower energies than with a proton target. In fact, the central area of heavy nuclei is "black", i.e. unitarity is saturated. As a result of saturation the transverse momentum distribution of gluons is modified. It gets a shape typical for the Cronin effect [53], i.e. gluons are suppressed at small, but enhanced at medium-large $p_{T}$. This effect is called color glass condensate (CGC) [55].

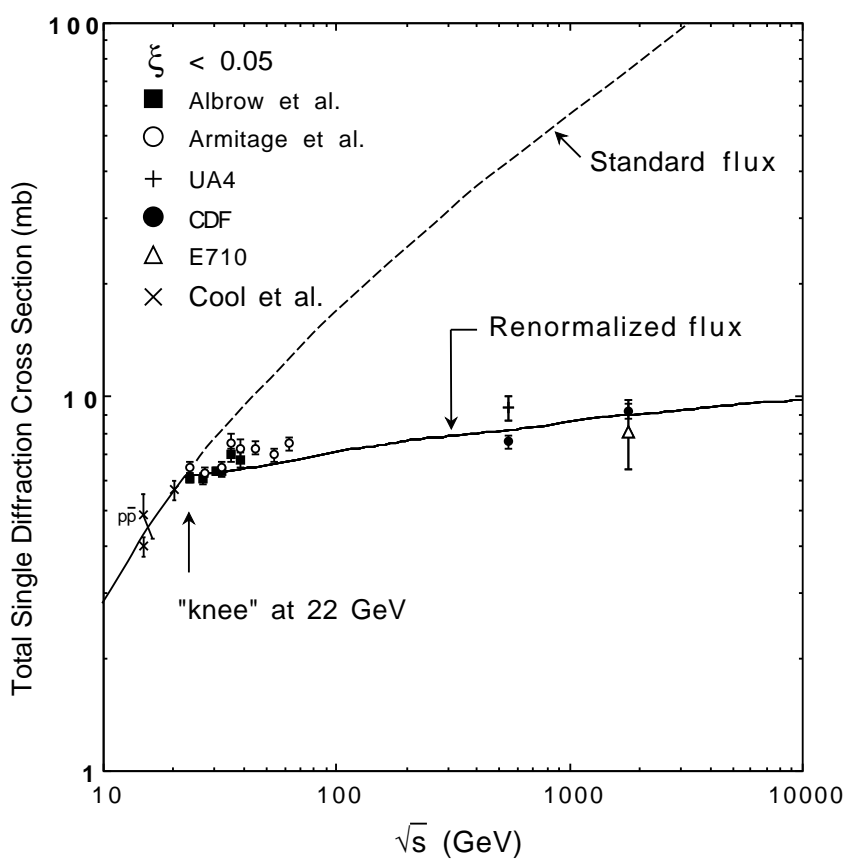

FIG. 15: Data for single diffraction in comparison with extrapolation of the nonunitarized Regge model (dashed curve) [43]

In the nuclear rest frame the same effect looks differently, as a color filtering of a glue-glue dipole [56]. Nuclear medium resolves dipoles of smaller size than in the case of a proton target. This results in increased transverse momenta of radiated gluons.

First theoretical observation of the CGC effect was made in diffraction in [57]. In a large rapidity gap process a dipole (e.g. a pion) propagates through the nucleus experiencing color filtering as is illustrated in Fig. 16. The nuclear matter

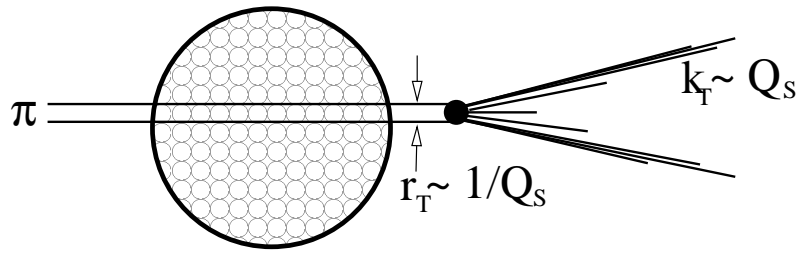

FIG. 16: Dipoles propagating through the nucleus experience color filtering leading to production of a di-jet with enhanced transverse momentum

is more transparent for small size dipoles having larger intrinsic momenta. The mean transverse momenta of quarks/jets rise $\propto R_{A}$. This is a direct measurement of the saturation scale which is expected to be

$$
Q_{s}^{2} \approx 0.1 \mathrm{GeV}^{2} A^{1 / 3} \approx 0.6 \mathrm{GeV}^{2} \quad \text { (for heavy nuclei) }
$$

This momentum is substantially larger than on a proton target. For gluon jets the saturation scale $Q_{s}^{2}$ should be doubled.

One may expect observation of real mini-jets at LHC. 


\section{CGC AND GLUON SHADOWING}

The CGC leads to a rearrangement of transverse momenta of gluons keeping their number unaltered [55]. However, interacting gluons not only push each other to higher transverse momenta, but also fuse resulting in reduction of gluon density. The latter effect is called shadowing. Both CGC and shadowing have the same origin: longitudinal overlap of gluon clouds originated from different bound nucleons. This is illustrated in Fig. 17. Bound nucleons in the nucleus do not overlap much,

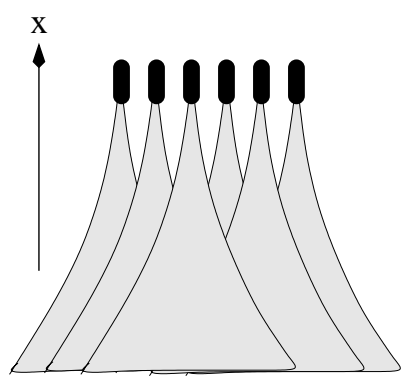

FIG. 17: Nucleons well separated in the longitudinal direction in the infinite momentum frame of the nucleus create gluonic fluctuations which overlap at small $x$

either in the rest frame, or in the infinite momentum frame, since both the nucleon size and internucleon spacing are subject to Lorentz contraction. However, gluons carrying a small fraction $x$ of the proton momentum have a smaller gammafactor and are less compressed in the longitudinal direction. Fig. 17 shows how gluonic clouds overlap at small $x$.

However, longitudinal overlap is not sufficient for gluon interaction, they must also overlap transversely. This may be a problem, since the transverse size of gluonic clouds is small. The mean number of overlapped clouds is,

$$
\left\langle n_{g}\right\rangle \approx \pi r_{0}^{2} \rho_{A} R_{A} \approx 0.3
$$

In this estimate we used the nuclear density $\rho_{A}=0.16 \mathrm{fm}^{-3}$, and the nuclear radius $R_{A}=7 \mathrm{fm}$.

Thus, according to (31) gluons have a rather small chance to overlap in impact parameters even in a nucleus as heavy as lead. Such a weak interaction of gluons leads to a weak gluon shadowing. Fig. 18 shows the results of calculations [9] for gluon shadowing in lead. The expected reduction of gluon density is less than $20 \%$ even at very small $x$. This was confirmed recently in [58] by an NLO analysis of NMC data for DIS on nuclei.

We conclude that smallness of gluonic spots in the proton prevents them from overlap in impact parameters even in heavy nuclei. This fact leads to substantial reduction of gluon shadowing and of the CGC effect compared to wide spread expectations.

\section{SUMMARY}

- Fock hadronic components which are eigenstates of interaction gain new weights in elastic scattering. The

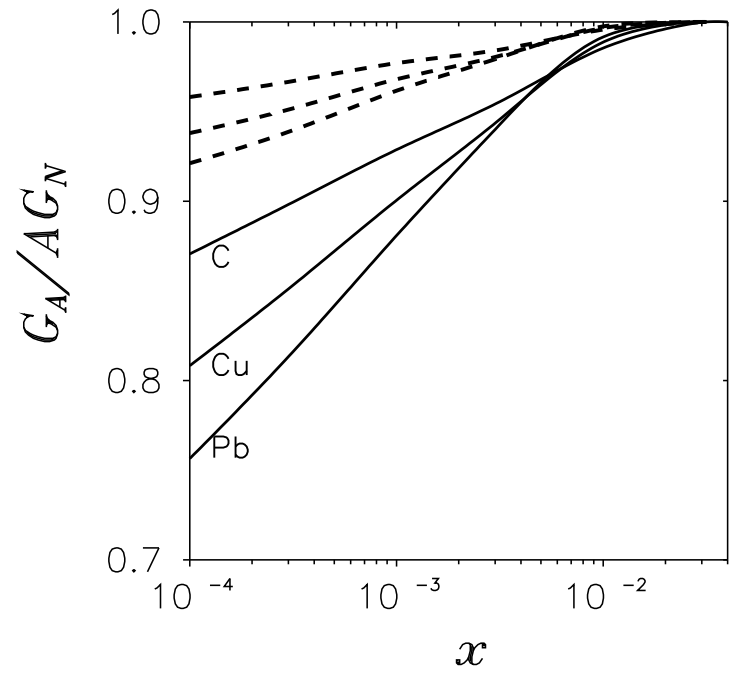

FIG. 18: Gluon shadowing, $G_{A} / G_{N}$ as function of Bjorken $x$ for carbon, copper and lead, at $Q^{2}=4 \mathrm{GeV}^{2}$ (solid curves) and $Q^{2}=$ $40 \mathrm{GeV}^{2}$ (dashed).

new composition formed by the interaction with the target can be projected to new states, thus diffractive excitation becomes possible.

- In QCD the eigenstates of the diffractive amplitude are color dipoles which preserve their size during interaction. Color transparency is the major effect governing diffraction.

- The observed puzzling smallness of high-mass diffraction related to diffractive gluon bremsstrahlung is a direct witness for the smallness of gluonic spots in hadrons. As a consequence, color transparency suppresses gluon radiation and gluon shadowing.

- Data on elastic scattering demonstrate an onset of the unitarity bound which causes strong breakdown of Regge factorization and suppresses diffraction. Indeed, a dramatic deviation from the Regge factorization has been observed in data. Asymptotically the fraction of diffraction vanishes as $1 / \ln (s)$.

- The observed shrinkage of diffractive cone in elastic $p p$ scattering originates mainly from onset of the unitarity bound, rather than from Gribov's diffusion of gluons. Data on $J / \Psi$ photoproduction confirm this demonstrating a weak shrinkage (in agreement with the predicted magnitude)

- Color filtering leads to a dramatic increase of transverse momenta of jets diffractively produced on nuclei. This would be a direct measurement of the saturation scale for the diffractive color glass condensate. 


\section{Acknowledgments}

One of us (B.K.) is grateful to Beatriz Gay Ducati for the kind invitation to I LAWHEP and many fruitful discussions.
This work was supported in part by Fondecyt (Chile) grants 1030355, 1050519 and 1050589, and by DFG (Germany) grant PI182/3-1.
[1] UA8 Collaboration, A. Brandt et al., Nucl. Phys. B 514, 3 (1998).

[2] Yu. L. Dokshitzer, V. A. Khoze, A. H. Mueller, and S. I. Troyan, Basics of Perturbative QCD, Editions Frontieres, ADAGP, Paris 1991

[3] E.A. Kuraev, L.N. Lipatov, and V.S. Fadin, Sov. Phys. JETP 44, 443 (1976); 45, 199 (1977); Ya.Ya. Balitskii and L.I. Lipatov, Sov. J. Nucl. Phys. 28, 822 (1978); L.N. Lipatov, Sov. Phys. JETP 63, 904 (1986).

[4] B.Z. Kopeliovich and I.P. Ivanov, Nucl. Phys. Proc. Suppl. B 146, 237 (2005).

[5] E. Feinberg and I.Ya. Pomeranchuk, Nuovo. Cimento. Suppl. 3, 652 (1956).

[6] M.L. Good and W.D. Walker, Phys. Rev. 120, 1857 (1960).

[7] B.Z. Kopeliovich, L.I. Lapidus, Pisma Zh. Eksp. Teor. Fiz. 28, 664 (1978).

[8] H.I. Miettinen and J. Pumplin, Phys. Rev. D 18, 1696 (1978).

[9] B.Z. Kopeliovich, A. Schäfer, and A.V. Tarasov, Phys. Rev. D 62, 054022 (2000)

[10] B.Z. Kopeliovich, L.I. Lapidus, and A.B. Zamolodchikov, Sov. Phys. JETP Lett. 33, 595 (1981); Pisma v Zh. Exper. Teor. Fiz. 33, 612 (1981).

[11] N.N. Nikolaev and B.G. Zakharov, Z. Phys. C 49, 607 (1991).

[12] J.M. Bjorken, J.B. Kogut, and D.E. Soper, D 3, 1382 (1971).

[13] K. Golec-Biernat and M. Wüsthoff, Phys. Rev. D 59, 014017 (1999).

[14] NE18 Collaboration, T.G. O’Neill, Phys. Lett. B 351, 87 (1995).

[15] A.S. Carroll et al., Phys. Rev. Lett. 61, 1698 (1988).

[16] B.K. Jennings and B.Z. Kopeliovich, Phys. Rev. Lett. 70, 3384 (1993).

[17] B.Z. Kopeliovich and J. Nemchik, Phys. Lett. B 368, 187 (1996).

[18] B.Z. Kopeliovich, J. Nemchick N.N. Nikolaev, and B.G. Zakharov, Phys. Lett. B 309, 179 (1993); Phys. Lett. B 324, 469 (1994).

[19] E665 Collaboration, M.R. Adams et al., Z. Phys. C 74, 237 (1997).

[20] HERMES Collaboration, A. Airapetian et al., Phys. Rev. Lett. 90, 052501 (2003).

[21] B.Z. Kopeliovich, J. Nemchik, A. Schafer, and A.V. Tarasov, Phys. Rev. C 65, 035201 (2002).

[22] B.Z. Kopeliovich, Habilitation thesis, LNPI, Leningrad, 1987

[23] E791 Collaboration, E.M. Aitala et al., Phys. Rev. Lett. 86, 4773 (2001).

[24] L. Frankfurt, G.A. Miller, and M. Strikman, Phys. Lett. B 304, 1 (1993).

[25] B.Z. Kopeliovich and B. Povh, Z. Phys. A 356, 467 (1997).

[26] M.B. Gay Ducati, V. P. Gonçalves, and M.V.T. Machado, Phys. Lett. B 506, 52 (2001).

[27] V.N. Gribov, Sov. Phys. JETP 56, 892 (1968).

[28] B.Z. Kopeliovich, J. Raufeisen, and A.V. Tarasov, Phys. Rev. C 62, 035204 (2000)

[29] B.Z. Kopeliovich Soft Component of Hard Reactions and Nu- clear Shadowing (DIS, Drell-Yan reaction, heavy quark production), in proc. of the Workshop 'Dynamical Properties of Hadrons in Nuclear Matter', Hirschegg 1995, ed. H. Feldmeier and W. Noerenberg, p. 102 (hep-ph/9609385).

[30] B.Z. Kopeliovich, A. Schäfer, and A.V. Tarasov, Phys. Rev. C 59, 1609 (1999).

[31] B.Z. Kopeliovich, J. Raufeisen, and A.V. Tarasov, Phys. Lett. B 503, 91 (2001)

[32] S.J. Brodsky, A. Hebecker, and E. Quack, Phys. Rev. D 55, 2584 (1997).

[33] M.A. Betemps, M.B. Gay Ducati, and M.V.T. Machado, Phys. Rev. D 66, 014018 (2002).

[34] M.B. Gay Ducati and M.V.T. Machado, Phys. Rev. D 65, 114019 (2002).

[35] B.Z. Kopeliovich, I. Schmidt, and A.V. Tarasov, paper in preparation.

[36] S.J. Brodsky, B. Kopeliovich, I. Schmidt, and J. Soffer, hep$\mathrm{ph} / 0603238$.

[37] R. Vogt and S.J. Brodsky, Nucl. Phys. B 478, 311 (1996).

[38] M. Franz, M. Polyakov, and K. Goeke, Phys. Rev. D 62, 074024 (2000).

[39] B.Z. Kopeliovich, Phys. Rev. C 68, 044906 (2003).

[40] B.Z. Kopeliovich, I.K. Potashnikova, and I. Schmidt, Phys. Rev. C 73, 034901 (2006).

[41] Yu.M. Kazarinov, B.Z. Kopeliovich, L.I. Lapidus, and I.K. Potashnikova, Sov. Phys. JETP 43, 598 (1976), Zh. Eksp. Teor. Fiz. 70, 1152 (1976).

[42] A.B. Kaidalov, Phys. Rept. 50, 157 (1979).

[43] K. Goulianos, Phys. Lett. B 358, 379 (1995).

[44] S. Erhan, P.E. Schlein, Phys. Lett. B481, 177 (2000).

[45] E.V. Shuryak, I. Zahed, Phys. Rev. D 69, 014011 (2004).

[46] M. D'Elia, A. Di Giacomo, and E. Meggiolaro, Phys. Lett. B 408, 315 (1997)

[47] B.Z. Kopeliovich, I.K. Potashnikova, B. Povh, and E. Predazzi, Phys. Rev. Lett. 85, 507 (2000); Phys. Rev. D 63054001 (2001).

[48] B.Z. Kopeliovich, Nucl. Phys. Proc. Suppl. A99, 29 (2001).

[49] B.Z. Kopeliovich and B. Povh, J. Phys. G30, S999 (2004).

[50] J. Hüfner, Y.P. Ivanov, B.Z. Kopeliovich, and A.V. Tarasov, Phys. Rev. D 62, 094022 (2000).

[51] ZEUS Collaboration, S. Chekanov et al., Eur. Phys. J. C24, 345 (2002).

[52] J. Hüfner and B.Z. Kopeliovich, Phys. Lett. B 426, 154 (1998).

[53] D. Antreasyan et al., Phys. Rev. D 19, 764 (1979).

[54] M.B. Gay Ducati, M.V.T. Machado, Phys. Rev. D 65, 114019 (2002).

[55] L. McLerran and R. Venugopalan, Phys. Rev. D 49, 2233 (1994); D 49, 3352 (1994).

[56] A.H. Mueller, Eur. Phys. J. A 1, 19 (1998).

[57] G. Bertsch, S.J. Brodsky, A.S. Goldhaber, and J.F. Gunion, Phys. Rev. Lett. 47, 297 (1981).

[58] D. de Florian and R. Sassot, Phys. Rev. D 69, 074028 (2004). 